\title{
Vibration energy harvesting: fabrication, miniaturisation and applications
}

\author{
S. P. Beeby*, D. Zhu \\ Electronics and Computer Science, University of Southampton, Highfield, Southampton, SO17 1BJ, \\ UK
}

\begin{abstract}
This paper reviews work at the University of Southampton and its spin-out company Perpetuum towards the use of vibration energy harvesting in real applications. Perpetuum have successfully demonstrated vibration-powered condition monitoring systems for rail and industrial applications. They have pursued applications were volume is not a particular constraint and therefore sufficient power can be harvested. Harvester reliability and longevity is a key requirement and this can be a challenging task in high shock environments. The University of Southampton has investigated the miniaturization of the technology. MEMS electromagnetic harvesters were found to be unsuitable although miniaturized devices fabricated using bulk components did perform well. Screen printed piezoelectric harvesters were also found to perform well and were ideally suited to a low profile application where device thickness was limited. Screen printing was not only used to deposit the active piezoelectric material but also an inertial mass ink based on tungsten. This enables the device to be printed entirely by screen printing providing a low-cost route to manufacture. Finally, details of a simulation tool that can take real world vibrations and estimate vibration energy harvester output was presented. This was used to simulate linear and nonlinear harvesters and in many applications with a characteristic resonant frequency the linear approach was found to be the optimum. Bistable nonlinear harvesters were found to work better with more random vibration sources.
\end{abstract}

Keywords: Vibration energy harvesting, electromagnetic energy harvesters, self-powered systems, piezoelectric thickfilms, linear vibration energy harvesters, nonlinear vibration energy harvester

\section{INTRODUCTION}

Vibration energy harvesting is the conversion of ambient kinetic energy, present in the form of vibrations, into electrical energy. It is an established research topic with numerous review papers on the topic as a whole ${ }^{1}$ and on more specific subtopics such as the use of piezoelectric materials ${ }^{2}$ and methods to increase the operational bandwidth of vibration energy harvesters ${ }^{3}$. Harvesting kinetic energy is just one of the sources of environmental energy that can be exploited, others including photovoltaic, thermoelectric and ambient $\mathrm{RF}^{4}$. Energy harvesting generally refers to the generation of electrical power in the microwatt to watt range and whilst the concept is identical to renewable power generation, the scale of the energy delivered, and some of the principles involved, is very different. Energy harvesting is typically used as a local power supply for autonomous systems such as wireless sensor nodes and therefore the harvester itself is just one part of a system that will usually include power management electronics, energy storage and the load electronics (i.e. the electronics required to perform the required function e.g. sensors, microprocessor and wireless communications) $)^{5}$. In order to optimize the system performance, the system design should be optimized as a whole and measures employed, such as duty cycling the system and careful selection of components, in order to minimize power consumption.

It is obvious the design of the load electronics is dictated by the requirements of the application, but the design of the vibration energy harvester ( $\mathrm{VEH})$ and its associated power conditioning electronics is also determined by the application. The characteristics of the environmental vibrations driving the VEH such as vibration amplitude, frequency spectra and any variations in environmental resonances will govern the dynamic behaviour of the harvester. Application constraints such as the space available for the harvester will influence the volume and form factor of the VEH. The required function of the load electronics will define the specification for the power output required from the VEH and the necessary capacity of the energy storage mechanism. Only by considering all these factors can the feasibility of a VEH to supply power for an autonomous system be determined.

This paper considers real applications of VEH and highlights the constraints imposed by applications and the lessons have been learnt in attempting to design a VEH within these constraints. The paper is a predominantly a review of work 
undertaken at the University of Southampton and at Perpetuum Ltd, a spin out company from Southampton that has developed practical VEHs and deployed autonomous systems powered by vibration energy harvesting. It also considers the fundamental effects of miniaturizing the technology and also the associated fabrication processes employed. The paper covers electromagnetic and piezoelectric energy harvesters fabricated using a variety of techniques and also discusses the effect of real application vibrations on the output of linear and nonlinear VEHs.

\section{VIBRATION ENERGY HARVESTING THEORY}

VEHs comprise of a mechanical system that couples environmental vibrations to a transduction mechanism that generates electrical energy from the motion of the mechanical system. A well designed mechanical system will maximize the energy coupled to the transduction mechanism and its behaviour may be linear or nonlinear. VEHs are typically inertial systems with the mechanical component (inertial mass) attached by a spring element to an inertial frame that acts as the fixed reference. The inertial frame transmits the vibrations to the suspended inertial mass producing a relative displacement between them. The size of the inertial mass, its amplitude and frequency of displacement determine the mechanical energy captured by the harvester. The vast majority of early VEHs were linear systems characterised by a traditional second order spring mass damper system. Such a system will possess a resonant frequency that should be designed to match a characteristic frequency present in the application environment. This approach magnifies the environmental vibration amplitude of by the quality factor (Q-factor) of the resonant system and this is an important consideration since vibration levels on the majority of industrial equipment are very low (discussed in more detail later). The theory of inertial based VEH is well documented ${ }^{6}$ and only the basic equations characterizing the mechanical energy captured by the harvester $\left(P_{a v}\right.$, equation 1$)$ and the power in the electrical domain $\left(P_{e}\right.$, equation 2$)$ are given below. In equation 1, the driving vibrations will determine the resonant frequency, $\omega_{\text {res }}$, of the VEH and the external vibration amplitude $Y$. The design of the VEH will determine the inertial mass, $m$, and the maximum inertial mass displacement, $z_{\max }$. Equation 1 also highlights the challenges associated with miniaturizing a VEH. The inertial mass scales with linear dimension $l^{3}$ and $z_{\max }$ scales with $l$ so $P_{a v}$ scales with $l^{4}$ and scaling devices down in size will fundamentally reduce the energy captured by the harvester. Miniaturization also tends to increase the resonant frequency and move $\omega_{\text {res }}$ away from the characteristic frequencies present in many potential applications.

$$
P_{a v}=\frac{m \omega_{r e s}^{3} Y z_{\max }}{2}
$$

Whilst equation 1 is informative, it is the power in the electrical domain which is of real interest and this is determined by the electrical and mechanical damping present. Electrical damping is provided by the transduction mechanism whilst mechanical damping refers to other losses such the effects of the surrounding air molecules and clamping losses. Maximum power occurs when these damping effects are equal and equation 2 can then be used to predict the power in the electrical domain where is $a$ the external acceleration and $Q_{O C}$ the open circuit quality factor of the VEH.

$$
P_{e}=\frac{m a^{2}}{8 \omega_{\text {res }}} Q_{O C}
$$

Equation 2 is a very useful equation since the variables can all be measured experimentally.

\section{PRACTICAL APPLICATIONS OF VIBRATION ENERGY HAREVSTING}

The first practical examples considered here come from Perpetuum's experience of applying the technology in industrial and rail applications ${ }^{7}$. In both cases the application is condition monitoring i.e. wirelessly monitoring the health of equipment and components. In both cases the VEH must supply sufficient power to sense, process and transmit vibration data that provides information regarding the health of, for example, a bearing. This is an elegant solution since the vibrations it is monitoring power the system. These scenarios provide a valuable insight into the technical challenges of the applications and the type of application scenario that makes a successful business case for vibration energy harvesting. 


\subsection{Rail applications}

The condition monitoring of rail wheel bearings is a very large potential market and includes both passenger and freight trains. There is a clear business argument for detecting the early deterioration in wheel bearings as failure in service can lead to expensive delays across the network and considerable customer inconvenience. In passenger trains the cost of retrofitting a wired solution is prohibitively expensive. In freight trains there is no power in any of the wagons so a wired solution is simply not an option. Batteries are not suitable due to the specified temperature range $\left(-40\right.$ to $\left.+85^{\circ} \mathrm{C}\right)$ and the additional maintenance requirement is not acceptable to the rail industry. Since the wheel bearings are located underneath the train, and given the dirty rail environment, solar cells are not an option. There are, however, high vibration levels but these are not at a fixed frequency and the VEH will also be subject to very high shock loading. Finally, there is a relatively large amount of free space available and the VEH is not constrained in this way.

Perpetuum have developed a complete sensor system enabled by energy harvesting. The VEH at the heart of the system has been carefully designed to operate within the required frequency range and survive very high shock levels. The system includes power management and energy storage and, together with the $\mathrm{VEH}$, this has all been designed to provide a 25 year maintenance free operational lifetime. The system can be simply bolted to existing bearing houses as shown in figure 1 and is therefore very easy to retrofit. The wireless sensor reports to a data collection module located elsewhere on the train and this in turn reports to the train operator via the cellular network providing an indication of bearing health updated very 15 minutes across the rail fleet.

It is interesting to contrast this application to the potential for vibration energy harvesting powered Tire Pressure Monitoring Systems (TPMS), which is a legal requirement in many countries. Tires provide high levels of vibration energy again with the potential for high amplitude shocks and there is a requirement for a maintenance free power supply but the business case is not so clear. The TPMS is periodically measuring and transmitting pressure data and not sampling accelerometers for sufficient time to gain an indication of bearing health, as is the case in the rail application. Therefore the TPMS requires considerably less energy to operate and batteries can supply the system for between 5 and 12 years. The automotive industry is also very cost sensitive and were a VEH to be developed that could replace the battery, it would have to be of comparable cost. This makes it an extremely challenging application that could potentially be addressed by a MEMS VEH ${ }^{8}$, but margins would be tight.
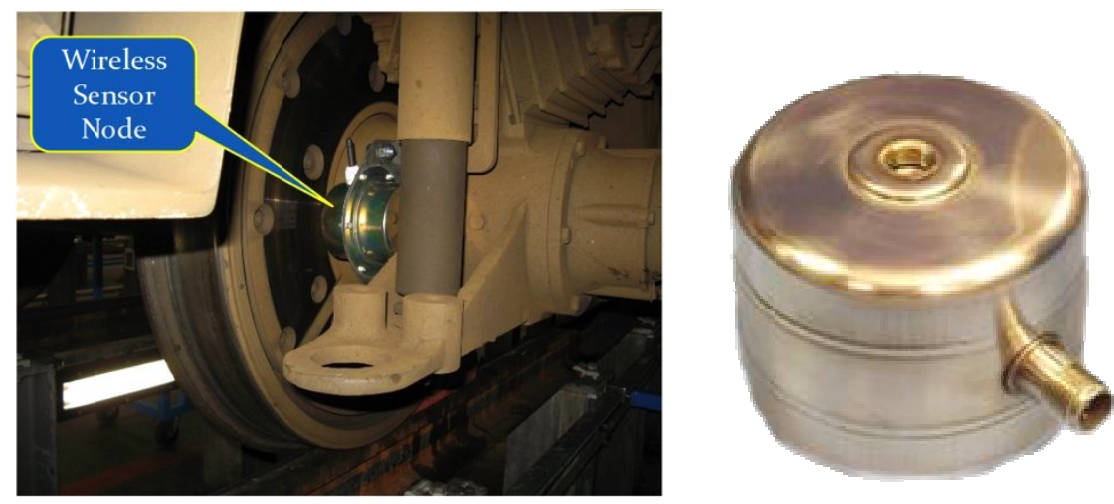

Figure 1. Perpetuum's VEH powered wireless wheel bearing monitoring system bolts to attaches to existing bolts enabling simple retrofitting (left). Perpetuum's industrial VEH (right).

\subsection{Industrial applications}

Perpetuum have also developed a VEH to power industrial condition monitoring equipment. Unlike the rail application, the majority of industrial applications operate at fixed frequencies $(50 / 60$ or $100 / 120 \mathrm{~Hz}$ - statistically the $100 / 120 \mathrm{~Hz}$ provides more power). This is the most straightforward case for a VEH since it can be designed to operate at a fixed resonant frequency. However, analysing vibration data in more detail reveals that in practice there is a range of frequencies and therefore the harvester bandwidth has to carefully chosen to capture as much of the range as possible. The vibration levels further complicate this, which can be very low $\left(<25 \mathrm{mg}, 1 \mathrm{~g}=9.81 \mathrm{~m} / \mathrm{s}^{2}\right)$. If the VEH bandwidth is too large, the Q factor may be too low to harvest sufficient energy from very low vibration amplitudes. Reliable operation is required over many years and the VEH must operate across wide temperature ranges. 
Perpetuum have developed a fully ATEX certified VEH that has been specified for use by the manufacturers of the leading industrial condition monitoring equipment (e.g. Emerson, GE Sensing, Honeywell). The power conditioning module developed by Perpetuum has been developed so it can also accept inputs from other types of energy harvesting modules (e.g. thermoelectric modules) enabling a range of harvesting solutions to be combined.

\section{MINATURISED ELECTROMAGNETIC VEH FOR INDUSTRIAL APPLICATIONS}

Perpetuum's solutions are fully engineered solutions for use where there are no real space constraints. Southampton's first work on a fixed frequency MEMS VEH was undertaken during the European Union (EU) funded project Vibration Energy Scavenging (VIBES) ${ }^{9}$. The project involved the miniaturization of electromagnetic ${ }^{10}$ and piezoelectric ${ }^{11}$ VEH for applications where space is more limited. The electromagnetic device, shown in figure 2, demonstrated many of the challenges associated with miniaturizing the technology using MEMS technology. The design places the coil (figure 2 (left) shows a traditional wound coil with internal diameter $0.6 \mathrm{~mm}$, outer diameter $2.4 \mathrm{~mm}, 390$ turns) on a silicon paddle that is designed to vibrate laterally (side to side). The magnetic field was supplied by bulk permanent magnets located in the packaging above and below the $5.7 \times 6 \times 0.525 \mathrm{~mm}$ chip. The power output from this device was just 0.12 $\mu \mathrm{W}$ at $3.4 \mathrm{~ms}^{-2}$ at $9.5 \mathrm{kHz}$ into $110 \mathrm{Ohm}$ load. This low power level was due to excessive mechanical damping and poor electromagnetic coupling. Also the frequency is far too high to be practically useful. Using an integrated coil in place of the wound coil (figure 2 right) makes the situation worse because of the increase in resistance of the coil ${ }^{12}$. This work confirms that electromagnetic devices of any type do not scale down in size favorably (certainly not to the microscale).

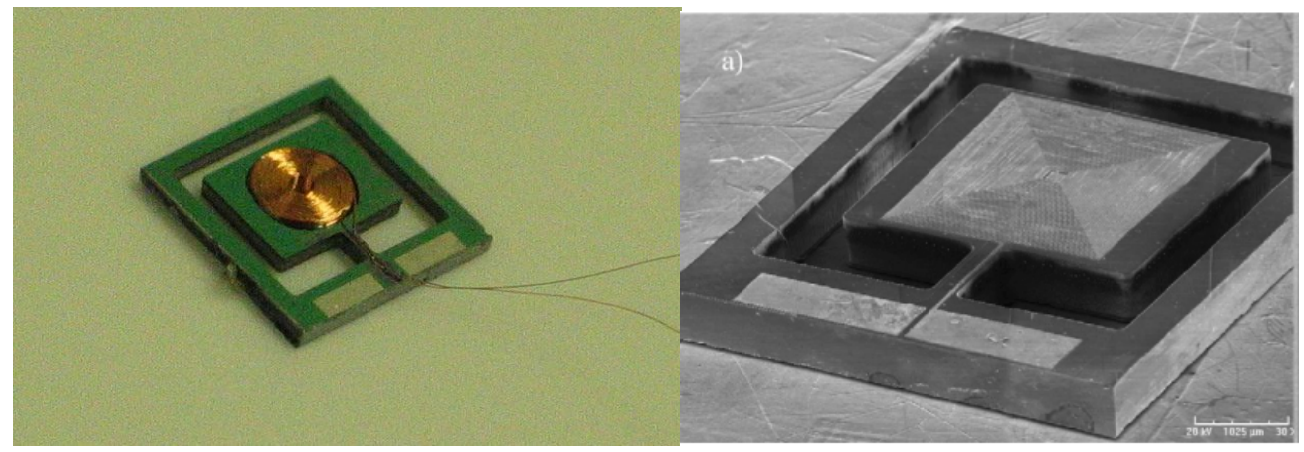

Figure 2. MEMS electromagnetic VEH with wound coil ${ }^{10}$ (left) and integrated coil ${ }^{13}$ (right)

The piezoelectric MEMS harvester shown in figure 3 was also fabricated in the VIBES project and this was more successful than the electromagnetic MEMS harvester with the integrated coil. It had an unpackaged device volume of 25 $\mathrm{mm}^{3}$ and delivered $1.4 \mu \mathrm{W}$ at $1.7 \mathrm{~V}$ from $19.6 \mathrm{~m} / \mathrm{s}^{2}$ acceleration at a frequency of $840 \mathrm{~Hz}^{11}$. It was found that an interdigital electrode arrangement produced the most power since it exploited the $\mathrm{d}_{33}$ piezoelectric properties of the film.

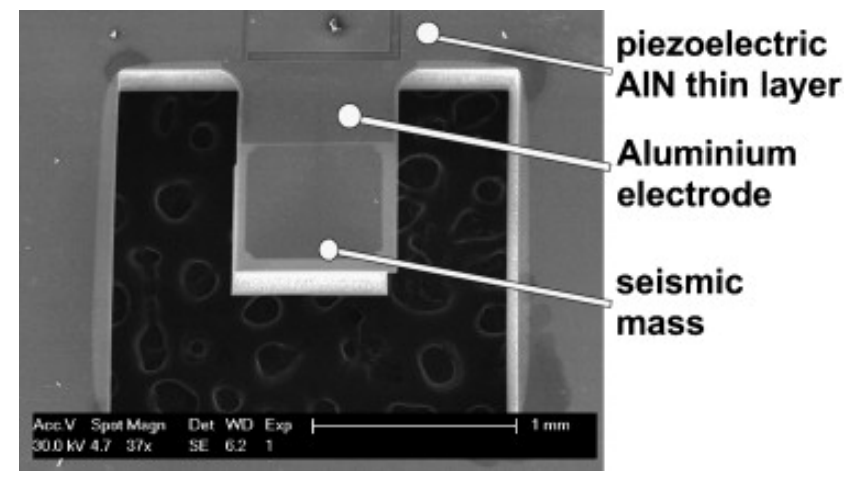

Figure 3. MEMS piezoelectric $\mathrm{VEH}^{11}$ 
Given the poor performance of the MEMS electromagnetic VEH, a miniature version was assembled by hand using bulk components. This represents a pragmatic approach for achieving a suitable electromagnetic coupling coefficient. The electromagnetic circuit was the same as for the MEMS device but the magnets were attached to a cantilever and moved relative to a fixed coil (see figure 4$)^{14}$. The VEH used a tungsten inertial mass and had a packaged size of $0.8 \mathrm{~cm}^{3}$ weighing 1.6 grams. Its power output was $58 \mu \mathrm{W}$, producing $1.12 \mathrm{~V}$ across a $15 \mathrm{k} \Omega$ load at an input acceleration of $0.6 \mathrm{~ms}^{-2}$. This equated to a conversion efficiency of $51 \%$ of mechanical energy converted into electrical. It was tuned to operate at $50 \mathrm{~Hz}$ matching the typical frequency of equipment using electrical motors and a condition monitoring system, shown in figure 4, was demonstrated powered entirely by this $\mathrm{VEH}^{15}$.
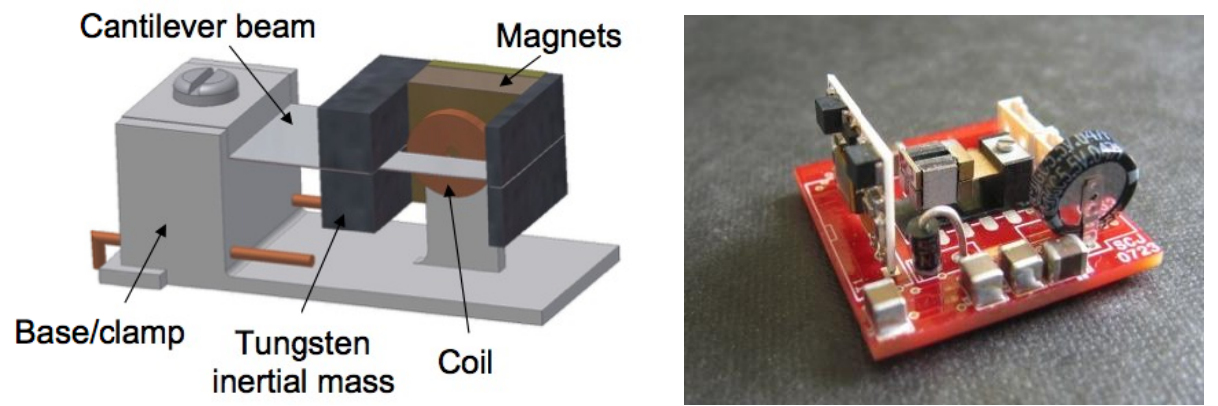

Figure 4. Miniature electromagnetic cantilever $\mathrm{VEH}^{14}$ (left) and self powered condition monitoring system ${ }^{15}$ (right).

\section{SCREEN PRINTED VEH FOR HELICOPTER APPLICATIONS}

The next example is another cantilever based piezoelectric VEH designed to harvest the vibrations experienced in a helicopter as part of an EU funded project TRIADE ${ }^{16}$. Helicopters are a good subject for vibration energy harvesting because the amplitude of vibrations are quite large across the airframe and the frequencies are fixed due to the constant rotor speed. The VEH was intended to power a system that monitored a range of parameters as part of a Health and Usage Monitoring System (HUMS). The challenge with this application was associated with the requirement to embed the system in the composite structure of the helicopter and therefore it has to be approximately the size of a credit card and $3 \mathrm{~mm}$ thick with a target power output for the VEH of $100 \mu \mathrm{W}$.

The solution developed was based upon a screen printed piezoelectric material ${ }^{17}$. Screen printing is an attractive and straightforward fabrication process for depositing active materials onto substrates and, by developing other screen printable materials such as a tungsten paste, the inertial mass can also be increased by the printing process. The cantilever was a T shape as shown in figure 5 with length $30 \mathrm{~mm}$, mass width $47 \mathrm{~mm}$ and a mass of 2.8 grams. The Tshape was used to minimize the tip displacement and it operated at around $66 \mathrm{~Hz}$ which matched a characteristic frequency of the helicopter. The power output was a very useful $240 \mu \mathrm{W}$ with a peak voltage of $5 \mathrm{~V}$ from an acceleration of $3.9 \mathrm{~ms}^{-2}$, which was sufficient to power the credit card sized system shown in figure 5 (right). This result was obtained on a shaker in the lab with a sinusoidal peak that matched the target application frequency. It was very interesting to observe that the output power falls when actual helicopter vibrations are replicated on the shaker rather than just a pure sine wave ${ }^{18}$. An investigation was also undertaken into the use of multiple piezoelectric layers by printing alternate electrode and piezoelectric layers ${ }^{19}$. Whilst this increases the surface area of the piezoelectric material, it also increases the device capacitance and the strain experienced will not be the same for each piezoelectric layer with the top most film experiencing maximum stress as the cantilever bends. It was found that two layers do increase the RMS power output by over $40 \%$ compared with the single layer. Adding a third layer, however, reduces the power by $20 \%$ compared with the two layer VEH. This is due to the uneven stress distribution and redistribution of the charge across all the electrodes. 

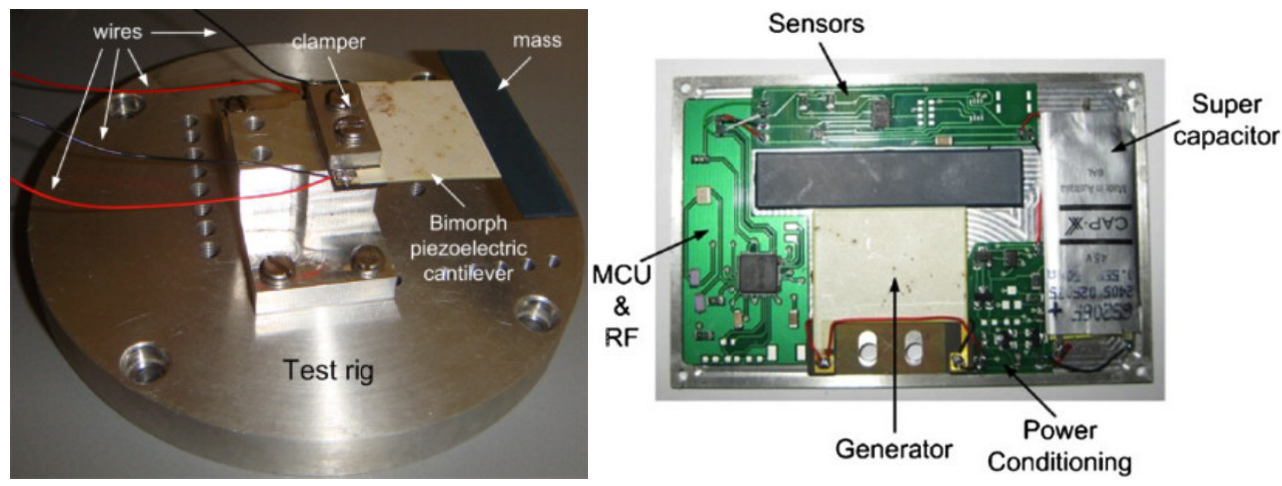

Figure 5. Screen printed piezoelectric VEH (left) and self powered system ${ }^{17}$ (right)

\section{APPLICATION OF REAL VIBRATION DATA TO LINEAR AND NONLINEAR VEH}

Two databases exist for real vibration data, one is held by the UK's energy harvesting network ${ }^{20}$ and the other held by the NiPS Laboratory at the University of Perugia ${ }^{21}$. These enable the user to download actual vibration data and replay them back through a laboratory shaker thereby testing devices with real application vibrations. Care should be taken to ensure the laboratory shaker is calibrated to compensate for any nonlinearities. This data has also been used in a C-based simulation tool to test the energy harvested by different types of $\mathrm{VEH}^{22}$. This work analysed eight types of VEH: linear electromagnetic with high Q, linear electromagnetic with low Q, electromagnetic bistable, electromagnetic Duffingnonlinear as well as linear piezoelectric with high Q, linear piezoelectric with low Q, piezoelectric bistable and piezoelectric Duffing-nonlinear. The output of these devices were simulated using vibration data from a ferry engine, a combined heat and power (CHP) plant pump, a car engine, a helicopter and white noise vibration. The purpose of this comparison was to compare linear, bistable and Duffing's nonlinear structure for each particular transducer. It is not the intention to compare the electromagnetic and piezoelectric transducers.

It was found that linear harvesters produce more energy in most applications where a clear characteristic frequency is present (e.g. ferry, CHP and helicopter). The Duffing non-linear produced more energy for a car engine due to its broader bandwidth. The bistable piezoelectric produced most energy from white noise. Electromagnetic bistable did not due to higher damping and lower velocities. This work presented a straightforward modelling tool that enables a particular type of VEH to be characterised against particular application scenarios. The results presented in the full paper used a specific set of parameters and these can all be adjusted in order to optimise a particular harvester design before subsequent fabrication.

\section{CONCLUSIONS}

Whilst fundamental vibration energy harvesting research is now mature, many research challenges exist in applying the technology and taking devices from the lab and out into the real world. In order to facilitate this, research should ideally consider real world applications at an early stage in order to ensure relevance and to appreciate the real world constraints. With vibration energy harvesting, size does matter: smaller devices produce less power for a given input excitation and also tend towards higher frequencies. The inherently low power of MEMS scale harvesters also weakens the business case for their use since a battery would last longer at these lower power levels. As well as optimising the transducer performance and mechanical design of the VEH, other ways of maximizing performance should not be overlooked. For example, increasing the inertial mass on MEMS harvesters will increase output power and lower operating frequency (e.g. a printed tungsten film of $120 \mu \mathrm{m}$ thick on a silicon $5 \times 5 \times 0.525$ structure would double its mass). Other types of VEH (e.g. bistable) may also provide more power in applications where the vibrations are more chaotic.

The criteria for a successful practical deployment of the technology are fairly obvious:

- Batteries and solar cells are unsuitable.

- Suitable vibrations present in the application environment (amplitude and frequencies). 
- Given any size constraints, the power output is sufficient for application electronics and operating scenario.

- Reliable VEH and system components.

- Development and implementation costs justified by user savings or other criteria (e.g. safety).

Commercializing the technology adds cost constraints that can radically affect harvester design. Design tools that incorporate real world vibrations are available that will assist in in assessing the viability of a particular application. Whilst there are not yet many large scale practical applications of the technology, the experiences of Perpetuum indicate they do exist and with the growing interest in topics such as the Internet of Things further opportunities will arise.

\section{REFERENCES}

[1] Beeby, S. P., Tudor, M. J. and White, N. M., "Energy harvesting vibration sources for microsystems applications," Meas. Sci. Technol. 17 (12), R175-R195 (2006).

[2] Sodano, H. A., Inman, D. J. and Park, G. "A review of power harvesting from vibration using piezoelectric materials," Shock and Vibration Digest 36(3), 197-206 (2004).

[3] Zhu, D., Tudor, J. and Beeby, S. "Strategies for increasing the operating frequency range of vibration energy harvesters: a review," Meas. Sci. and Technol. 21 (2), 022001 (2010).

[4] Vullers, R. J. M., van Schaijk, R., Doms, I., Van Hoof, C. and Mertens, R. "Micropower energy harvesting,” SolidState Electronics 53(7), 684-693 (2009).

[5] Beeby, S. and White, N., Eds. [Energy Harvesting for Autonomous Systems], Artech House, Inc., Norwood MA (2009).

[6] Stephen, N. G. “On energy harvesting from ambient vibration,” Journal of sound and vibration, 293(1), 409-425 (2006).

[7] Perpetuum webpage $<$ http://www.perpetuum.com/> (27 March 2015).

[8] Vullers, R. J. M., Renaud, M., Elfrink, R. and van Schaijk, R. "MEMS based vibration harvesting: Facing the ugly

facts," Solid-State Sensors, Actuators and Microsystems (TRANSDUCERS \& EUROSENSORS XXVII), 2013

Transducers \& Eurosensors XXVII: The 17th International Conference on, 685,688, (2013).

[9] VIBES project website < http://www.vibes.ecs.soton.ac.uk/> (27 March 2015).

[10] Beeby, S. P., Tudor, M. J., Koukharenko, E., White, N. M., O'Donnell, T., Saha, C., Kulkarni, S. and Roy, S.

"Design and performance of a microelectromagnetic vibration powered generator," In Solid-State Sensors, Actuators and Microsystems Digest of Technical Papers 1, 780-783 (2005).

[11] Marzencki, M, Ammar, Y. and Basrour S. "Integrated power harvesting system including a MEMS generator and a power management circuit," Sensors and Actuators A: Physical 145-146, 363-370 (2008).

[12] O’Donnell, T., Saha, C., Beeby, S. and Tudor, J. "Scaling effects for electromagnetic vibrational power generators," Microsystem Technologies 13(11-12), 1637-1645 (2007).

[13] Kulkarni, S., Koukharenko, E., Torah, R., Tudor, J., Beeby, S., O’Donnell, T. and Roy S. "Design, fabrication and test of integrated micro-scale vibration-based electromagnetic generator," Sensors and Actuators A: Physical 145-146, Pages 336-342 (2008).

[14] Beeby, S. P., Torah, R. N., Tudor, M. J., Glynne-Jones, P., O'Donnell, T., Saha, C. R. and Roy, S. “A micro electromagnetic generator for vibration energy harvesting," Journal of Micromechanics and Microengineering 17(7), 1257-1265 (2007).

[15] Torah, R., Glynne-Jones, P., Tudor, M., O'Donnell, T., Roy, S. and Beeby, S. "Self-powered autonomous wireless sensor node using vibration energy harvesting," Measurement Science and Technology 19(12), 125202 (2008).

[16] Project webpage "Development of Technology Building Blocks for Structural Health-Monitoring Sensing Devices In Aeronautics" <http://ec.europa.eu/research/transport/projects/items/triade_en.htm> (27 March 2015).

[17] Zhu, D., Beeby, S. P., Tudor, M. J., Harris, N. R. “A credit card sized self powered smart sensor node," Sensors and Actuators A: Physical 169 (2), 317-325 (2011).

[18] Zhu, D., Beeby, S., Tudor, J., Grabham, N., White, N. and Harris, N. "Performance of a piezoelectric energy harvester under vibrations taken from a helicopter," Technical Digest of PowerMEMS 2011, 221 - 224 (2011). 
[19] Zhu, D., Beeby, S., Tudor, J., White, N. and Harris, N. "Improving output power of piezoelectric energy harvesters using multilayer structures," Procedia Engineering, 25, 199-202 (2011).

[20] EH Network Data Repository < http://eh-network.org/data/> (27 March 2015).

[21] Real Vibrations database <http://realvibrations.nipslab.org/home> (27 March 2015).

[22] Beeby, S. P., Wang, L., Zhu, D., Weddell, A. S., Merrett, G. V., Stark, B., Szarka, G. and Al-Hashimi, B. M. “A comparison of power output from linear and non-linear kinetic energy harvesters using real vibration data," Smart Materials and Structures, 22, (7), 075022 (2013). 